\title{
Layer-by-layer deposition of functional click polymers for microarray applications
}

\author{
L. Sola , A. Romanato, M. B. Siboni, F. Damin, E. Chiodi, D. Brambilla, M. Cretich, A. Gori, \\ M. Chiari
}

Institute of Chemistry of Molecular Recognition, National Research Council of Italy, Via Mario Bianco 9, 20131 Milan, Italy

Received 13 March 2019; accepted in revised form 18 June 2019

\begin{abstract}
Bioprobes immobilization methods that elevate the probes from the substrate are generally preferred in microarray technology because they prevent steric limitations during the hybridization of the target to probes. A versatile approach to control the thickness of a polymeric coating based on click chemistry to obtain covalently linked layer-by-layer coatings for surface functionalization is presented. By alternating cycles of coating using copolymers bearing click groups, the thickness of the film increases, while remaining functional and stable. Click chemistry reactions provide numerous advantages over standard conjugation procedures typically used in microarrays. They include: quantitative yields and insensitivity of the reaction to $\mathrm{pH}$ and hydrolysis. Moreover, click reactions do not interfere with organic groups naturally present in DNA, proteins and peptides such as amino and carboxyl groups allowing orthogonal and chemoselective probe immobilization. In addition to the formation of multilayers, click reactions allow to bind biomolecules to polymer chains generating so-called polymeric probes, which are then immobilized on microarray supports. In a microarray assay of clinical relevance, this methodology provides a miniaturized, tri-dimensional multilayer with higher density of capture probe, improved hybridization efficiency and sensitivity.
\end{abstract}

Keywords: coatings, click chemistry, layer-by-layer coating, localized surface functionalization, microarray

\section{Introduction}

Microarray technology is a powerful analytical technique widely exploited to study interactions between biomolecules that has recently found great applications in the development of diagnostic platforms for clinical practice. The success of the technique in diagnostics depends, among other factors, on the robustness of the coupling reaction between the probe and the surface. The most important parameters that influence molecular recognition include: the physical-chemical properties of the surface, which in turn, influence specific and non-specific binding of both target and non-target biomolecules; the distance between the chip surface and the immobilized probes; the density of the immobilized probe, which determines the limit of detection and sensitivity of the analytical system, and finally the orientation of the tethered molecules, that might facilitate the target binding, or, in some cases impair it, especially when considering large analytes such as proteins $[1,2]$. The coating of microarray supports with thin films is one of the best methods to improve the surface properties and to control the interactions between the probe and the target. Among several techniques exploited to modify microarray surfaces, polymeric coatings lead to formation of homogenous coatings that expose functional groups in the axial position, that means away from the surface, facilitating the biomolecular recognition between probe and target. Our group has devoted a lot of efforts to the development of tailored coatings for biosensing applications. In particular, a copolymer obtained by free

$\overline{{ }^{*} \text { Corresponding author, e-mail: laura.sola@icrm.cnr.it }}$

(C) BME-PT 
random radical polymerization of $N, N$-dimethylacrylamide (DMA) revealed to be an ideal candidate for probe immobilization [3]. This copolymer, named copoly(DMA-NAS-MAPS) easily forms a thin film on the surface of different materials [4] by a combination of physi- and chemisorption, and contains succinimidyl esters and trimethoxysilane moieties pending from the polymer backbone, that confer functionalities and stability onto the coated surfaces. More recently, starting from this common precursor a family of polymeric coatings with different functionalities has been obtained by post polymerization modification (PPM) of the succinimidyl esters with a proper bifunctional amine [5]. In post-polymerization modification, monomers bearing chemoselective handles that are inert towards the polymerization conditions are first polymerized and then converted into a broad range of other functional groups [6-8]. This method has gained increasing attention over the past years as it allows to introduce functional groups incompatible with polymerization conditions. In particular, versatile functional groups that enable the so called click reactions were introduced: azide, alkyne and dibenzocyclooctine (DBCO). Figure 1 reports the chemical structure of polymer precursor and derivatives.

In this work, we exploit the characteristics of the copolymers combined with the presence of click groups and silanols in their backbone to easily obtain multilayered coatings and 3D-coatings with different

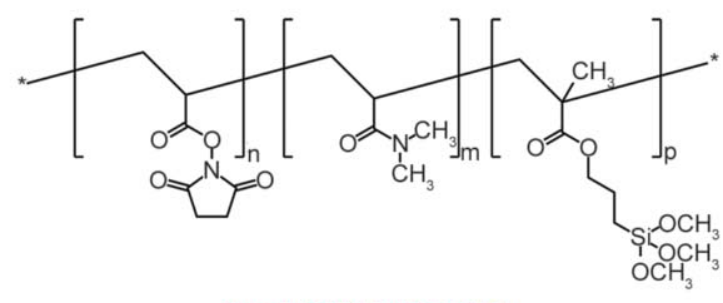

Copoly (DMA-NAS-MAPS)

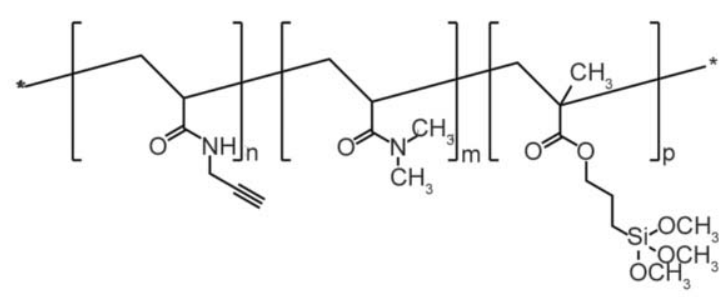

Copoly alkine architectures. The multilayers formed by a combination of a covalent reaction between 'clickable' functionalities and silanol condensation, increase the thickness of the coating, facilitating the recognition by the target, since the immobilized probe is better exposed on the surface [9].

Usually, multilayered coatings are obtained by a method defined layer-by-layer (LbL) deposition, a versatile technique for fabricating tailored thin films by either electrostatic or hydrogen bonding interactions $[10,11]$. More recent works have reported polymer multilayer assembly facilitated by covalent bonding $[12,13]$. Covalently bound films offer the advantage of higher stability due to the cross-linking between polymer networks and are not susceptible to disassembly under varying solution conditions (e.g., salt, pH), as it is typically observed for a range of electrostatically coupled and H-bonded films. Herein, we report a highly efficient and generalizable method, based on click chemistry and silanol condensation, to construct LbL functional polymer films. In particular, the substrate undergoes repeated cycles of coating, using copolymers with complementary click functionalities (see scheme in Figure 2). Each step consists of a quick immersion in a polymer solution followed by rinse and dry steps. A similar approach was exploited by Yang et al. [14] to obtain a multilayer coatings with anti-biofouling properties. Similarly, Such et al. [15] proposed a strategy, based on click chemistry, to assembly layer by layer
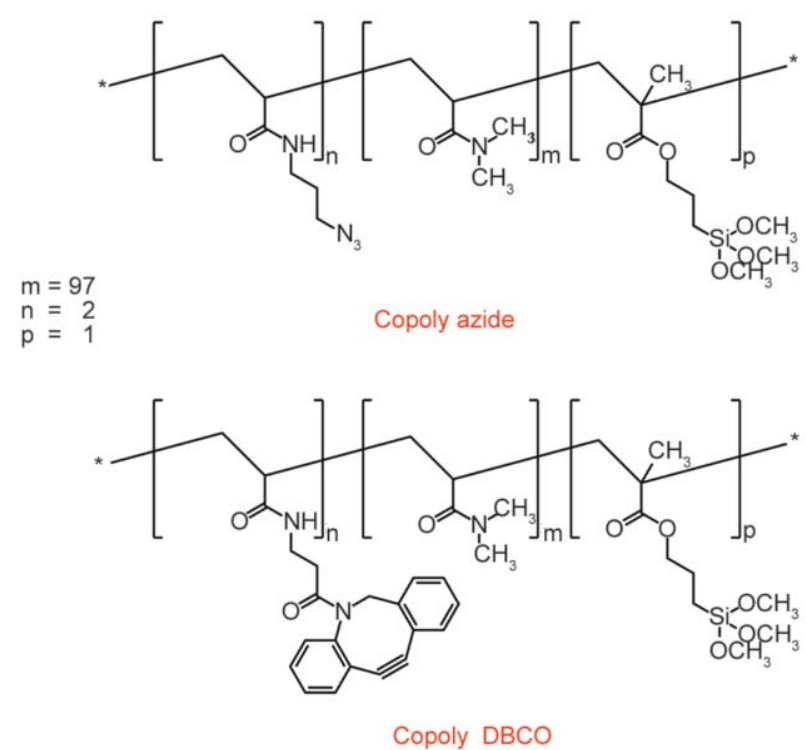

Figure 1. Chemical formulas of the click copolymers used in this study. The copolymers were obtained by post-polymerization modification of a common precursor, synthesized by random free radical polymerization of the monomers, mixed in the indicated molar percentage. 

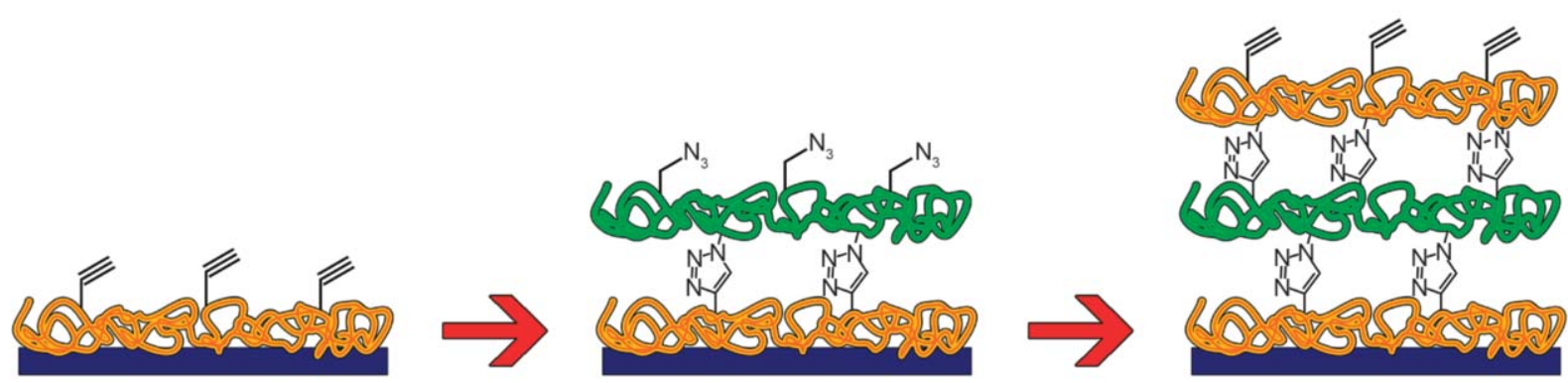

Figure 2. Scheme of the formation of multilayers. A first coating with copoly alkyne is performed on a silicon oxide slide. Then a second coating is layered on top of the first one and they react covalently through the click chemistry reaction between triple bonds and azide groups. Finally a third layer with copoly alkyne is obtained.

poly(acrylic acid) coatings with controlled thickness. However, to the best of our knowledge, none of the previous works combine click reaction and silanol condensation. In addition, the existing multilayer coatings were never applied to microarray technology. The copolymers introduced by our groups have already revealed optimal characteristics to be used as coatings for microarrays, since they combine the presence of functionalities to covalently bind biomolecules, together with reduced surface charges and anti-fouling properties which result in a minimal background signal.

In another configuration, we exploit the non-reactivity of click functions to hydrolysis not only to increase the thickness of the film, but also to modify the bioprobes in order to mediate their immobilization onto surfaces with functionalities that, otherwise, would be incompatible. Multiplexing of the probes is the main characteristic of microarray technology, however all the probes must have the same functionality to be able to react with the substrate. Binding the probe to the polymer before spotting allows to immobilize biomolecules with different functional groups on the same surface. For example, azido or DBCO biomolecules (DNA, peptides or antibodies) are linked to polymer chains through click reactions generating what we call a polymeric probe, which is then immobilized on microarray supports, coated with complementary functionalities (see Figure 3). In this case an azido-modified biomolecule

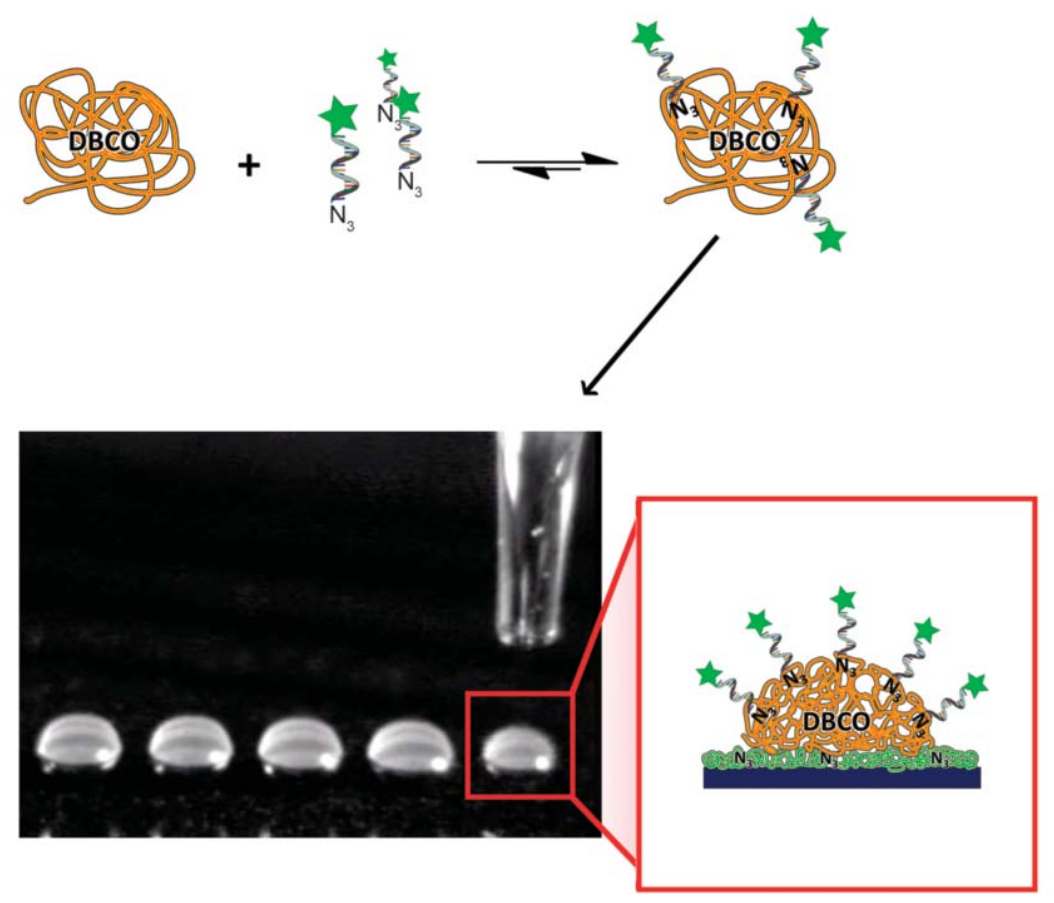

Figure 3. Schematic representation of the formation of a polymeric probe: a solution of copoly DBCO is incubated with the azido-modified oligonucleotide to obtain a polymeric probe that is then spotted on a surface coated with copoly azide. The azide modified oligonucleotide can bind to the surface thanks to the presence of DBCO groups of the polymer chains. 
can be bound to an azido modified polymers since the binding with the surface is mediated by the DBCO groups of the polymer to which the azido probe is bound. This strategy not only offers the possibility of immobilizing probe with functionalities incompatible with those on the surface, but also generates a sort of tri-dimensional structure with higher functionality density, thus increasing the quantity of probe immobilized on the surface. Moreover, this strategy results in a miniaturized multilayer with a reduced functionalized area, a very important feature for applications on micro-dimensional platforms and biosensors.

\section{Materials and methods}

$N, N$-Dimethylacrylamide (DMA), 3-(trimethoxylsilyl)propyl methacrylate (MAPS), dibenzocyclooctyne-amine, anhydrous tetrahydrofuran (THF), ammonium sulfate $\left[\left(\mathrm{NH}_{4}\right)_{2} \mathrm{SO}_{4}\right]$, phosphate-buffered saline (PBS), ethanolamine, were purchased from Sigma Aldrich (St. Louis, MO, USA). 3-azido-1propylamine was synthesized as reported elsewhere [16]. All oligonucleotides for hybridization tests were synthesized by Metabion International AG (Germany) with the following sequences:

5'-GCCCACCTATAAGGTAAAAGTGA-3'

[COCU8; modified in $5^{\prime}$ with the following functionalities: amine $\left(\mathrm{NH}_{2}\right)$, dibenzocyclooctyne (DBCO), and azide $\left(\mathrm{N}_{3}\right)$,

5'-Cy3-TCACTTTTACCTTATAGGTGGGC-3'

(COCU10), labelled with the fluorophore cyanine3 (Cy3) for fluorescence detection; COCU11 has the same sequence of COCU10, but was labelled with $\mathrm{Cy} 3$ in $5^{\prime}$ and modified with DBCO or $\mathrm{N}_{3}$ in $3^{\prime}$. These oligonucleotides were freeze-dried and suspended in DI water at a final concentration of $100 \mu \mathrm{M}$ before use.

Peptides OMPA bearing azido or DBCO groups were synthesized as reported in [17], by stepwise microwave-assisted Fmoc-SPPS on a Biotage ALSTRA Initiator + peptide synthesizer according to well-established protocols. Briefly, peptides were assembled on a 2-CTC resin. Chain elongation was performed by iterative cycles of amino acids coupling (using Oxyma/DIC as activators) and Fmoc-deprotection using a $20 \%$ piperidine solution in DMF. Upon complete chain assembly, peptides were cleaved from the resin using a $2.5 \%$ TIS, $2.5 \%$ thioanisole, $2.5 \%$ water, $92.5 \%$ TFA mixture. Crude peptides were then purified by preparative RP-HPLC. MS analysis was performed separately on purified material. The chromatographic columns were from Phenomenex (Torrance CA, USA). OMPA-DBCo peptide For OMPA-DBCO the calculated mass was $[\mathrm{M}+1]+=2814.8,[\mathrm{M}+2] 2+=1407.9,[\mathrm{M}+3] 3+=$ 938.9; Mass found by $(\mathrm{ESI})$ was $[\mathrm{M}+1]+=2814.2$, $[\mathrm{M}+2] 2+=1407.6,[\mathrm{M}+3] 3+=938.8$. For OMPA$\mathrm{N} 3$ peptide calculated mass was $[\mathrm{M}+1]+=3335.6$, $[\mathrm{M}+2] 2+=1668.3,[\mathrm{M}+3] 3+=1112.5,[\mathrm{M}+4] 4+=$ 834.7; Mass found by (ESI): $[\mathrm{M}+2] 2+=1668.4$, $[\mathrm{M}+3] 3+=1112.5$.

Polyclonal rabbit antibody against OMPA peptide was purchased from PRIMM s.r.l. (Milan, Italy). Untreated silicon slides $1000 \AA$ thermal oxide $\left(14 \times 14 \mathrm{~mm}^{2}\right)$ were supplied by Silicon Valley Microelectronics Inc. (Santa Clara, CA, USA) and were pretreated using a Harrick Plasma Cleaner, PDC-002 (Ithaca, NY, USA) connected to an oxygen line.

Dual Polarization Interferometry (DPI) analyses were conducted using an Analight Bio 200 (Farfield Group, Biolin Scientific, Manchester, UK) supporting the Analight Explorer software.

Spotting was performed using SciFLEXARRAYER S12 (Scenion, Berlin, German). Fluorescence images were obtained using a ScanArrayLite and data were analyzed using the ScanArray Express software, both by Perkin Elmer (Waltham, MA, USA).

\subsection{Polymer synthesis}

Click copolymers have been obtained by post polymerization reaction of the parent polymer copoly (DMA-NAS-MAPS), which is composed of DMA (97\% mole percent), $N$-succinimidyl acrylate (NAS) (2\% mole percent), and 3-(trimethoxysilyl)propyl methacrylate (MAPS, 1\% mole percent) [5]. The polymer was synthesized by free radical polymerization as reported elsewhere [18]. Briefly, after degassing anhydrous THF with helium, DMA, NAS, and MAPS were added to the reaction flask so that the total monomer feed was $20 \% \mathrm{w} / \mathrm{v}$. The reaction mixture was heated to $65^{\circ} \mathrm{C}$ for two hours in the presence of AIBN. The crude material was cooled to room temperature and diluted 1:1 with dry THF; the solution was then precipitated in petroleum ether (10 times the volume of the reaction mixture) to eliminate unreacted monomers.

This succinimidyl copolymer was modified by reaction with 3-azido-1-propylamine and dibenzocyclooctyne-amine. To introduce the new functionalities, a $20 \% \mathrm{w} / \mathrm{v}$ solution of the copolymer was prepared by dissolving it in dry THF and a 2.5 molar excess with 
respect to the moles of NAS of the proper amine was added to the crude material, assuming that the concentration of NAS along the polymer chain is $40 \mathrm{mM}$ [5]. The mixture was stirred for $5 \mathrm{~h}$ at room temperature and then diluted 1:1 with anhydrous THF. The polymers were precipitated in petroleum ether (10 times the volume of the reaction mixture), filtered on a Büchner funnel, and dried under vacuum at room temperature. To further purify the powder obtained, the polymers were dissolved again in anhydrous THF to a final concentration of $10 \% \mathrm{w} / \mathrm{v}$ and re-precipitated in petroleum ether. The powder was finally filtered and dried again under vacuum at room temperature.

\subsection{Polymer size determination}

The size of copoly(DMA-NAS-MAPS) was characterized using Gel Permeation Chromatography in tandem with multi-angle laser light scattering (MALLS). An Agilent 1200 series liquid chromatography system, consisting of an isocratic pump and auto sampler is connected upstream of both the GPC-MALLS systems to control mobile phase flow throughout each system. Agilent ChemStation software was used to control the sequence of sample injection and flow rates.

The GPC setup consists of four Shodex aqueous GPC columns in series: OHpak SB-G (guard column), OHpak SB-804 M HQ, OHpak SB-803 HQ, and OHpak SB-802.5 HQ. Each column is packed with a polyhydroxymethacrylate gel and connected in series with a decreasing exclusion limit. The columns were maintained at $25^{\circ} \mathrm{C}$ throughout each run using an Agilent 1200 thermostatic column compartment. After the polymer sample is fractionated by GPC, the sample flows into a DAWN HELEOS II (Wyatt Technology, Santa Barbara, CA, USA), which detects scattered light at 18 angles. The light scattering data obtained by the DAWN HELEOS II is used to measure the radius as well as the absolute molecular weight of the sample in solution. The MALLS instrument is in-line with an Optilab rEX (Wyatt Technology) refractive index detector, which measures the change in refractive index of the fractionated sample. ASTRA software (ver. 5.3.4, Wyatt Technology) was used to analyze the light scattering and refractive index data. The data was fit with a $2^{\text {nd }}$ order Berry model and the $\mathrm{d} n / \mathrm{d} c$ values of each run were calculated by assuming $100 \%$ mass recovery through the columns.
Dry poly(DMA-NAS-MAPS) was diluted using the GPC mobile phase (GPC buffer: $100 \mathrm{mM} \mathrm{NaCl}, 50$ $\mathrm{mM} \mathrm{NaH}_{2} \mathrm{PO}_{4}$, and $200 \mathrm{ppm} \mathrm{NaN}$ ) to a concentration of $2.66 \mathrm{mg} / \mathrm{ml}$ and the sample was run three times through the GPC-MALLS system to test for reproducibility. Each run injected $100 \mu \mathrm{l}$ of sample to be analyzed and the flow rate through the system was held at a constant $0.3 \mathrm{ml} / \mathrm{min}$. The $M_{\mathrm{w}}$ of poly (DMA-NAS-MAPS) by GPC-MALLS was found to be $3.6 \cdot 10^{4} \mathrm{~g} / \mathrm{mol}$ with a polydispersity of 2.4 .

\subsection{C13-NMR characterization of azide and DBCO polymers}

C-13 NMR spectra were acquired using 500 Bruker DMX and 600 Bruker DRX spectrometers equipped with $5 \mathrm{~mm}$ TXI probe with $z$ gradient at room temperature $(300 \mathrm{~K}$. Approximately $30 \mathrm{mg}$ of polymer was dissolved in DMSO- $d_{6}$ solvent. Spectra were calibrated on the DMSO solvent signal at $40.45 \mathrm{ppm}$. ${ }^{13} \mathrm{C}$ NMR (DMSO), $\delta$ [ppm]: 174.6 (backbone carbonyl), 166 (succinimide carbonyl) 40-30 (methylene carbons). The degree of succinimide insertion was determined from the ratio of the integrals of backbone and succinimide carbons, and the NAS molar fraction was found to be 0.015 . Literature data suggest that the apparent reactivity ratios obtained for the DMA/NAS copolymerization indicate that the reactivity of a macro radical terminated either by a DMA unit or by a NAS unit towards the other monomer is similar: in both cases, cross-propagation is favored over homo-propagation [19].

NMR spectra of post polymerization modified polymers were compared to the spectrum of the parent polymer to assess the insertion of the new functionalities as well as the disappearance of the succinimide carbonyl signal. In the carbonyl region, between 160 and $180 \mathrm{ppm}$ a new signal appears at $173.8 \mathrm{ppm}$ in both, azide and DBCO modified polymers, and it is attributed to the new amido group formed between the amine of the substituents and the backbone. In the region between 120 and $135 \mathrm{ppm}$, the DBCO aromatic signals are clearly detected, whereas between $48-50$ and $39 \mathrm{ppm}$, the azido signals appears.

\subsection{Surface coating}

\subsubsection{Single layer coating}

Silicon oxide slides were pre-treated with oxygen plasma for $10 \mathrm{~min}$ : the oxygen pressure was set to 1.2 bar with a power of $29.6 \mathrm{~W}$. Each copolymer was 
dissolved in DI water to a final concentration of $2 \% \mathrm{w} / \mathrm{v}$ and then diluted $1: 1$ with a solution of ammonium sulphate $1.6 \mathrm{M}$. The slides were immersed into each solution for $30 \mathrm{~min}$, then rinsed with DI water, dried under a nitrogen stream, and finally cured under vacuum at $80^{\circ} \mathrm{C}$ for 15 minutes.

\subsubsection{Multilayer formation}

Silicon chips, pre-treated and coated with copoly azide as described above, were immersed overnight in a $1 \% \mathrm{w} / \mathrm{v}$ water solution of copoly DBCO. After each step, the slides were rinsed with DI water, dried under nitrogen stream and cured at $80^{\circ} \mathrm{C}$ for 15 minutes. Subsequently the slides were immersed again in a $1 \% \mathrm{w} / \mathrm{v}$ water solution of copoly azide.

The coated slides were spotted with oligonucleotides labelled in 5' position with $\mathrm{Cy} 3$ for fluorescence detection and in $3^{\prime}$ position with an amine, $\mathrm{DBCO}$ or azide modification (COCU11). The oligonucleotides were diluted to $10 \mu \mathrm{M}$ in $150 \mathrm{mM}$ phosphate buffer containing $0.01 \% \mathrm{w} / \mathrm{v}$ of sucrose monolaurate. The oligonucleotides were deposited onto the surface using a noncontact microarray spotter (Scienion sciFLEXARRAYER S12) using a $80 \mu \mathrm{m}$ nozzle. The spot volume, temperature, and humidity were precisely controlled to $400 \mathrm{pl}, 22^{\circ} \mathrm{C}$, and $65 \%$, respectively; spot pitch was set at $350 \mu \mathrm{m}$. Immediately after spotting, all chips were stored overnight in a sealed chamber, saturated with sodium chloride ( $40 \mathrm{~g} / 100 \mathrm{ml} \mathrm{H}_{2} \mathrm{O}$ ). All chips were then rinsed with DI water and immersed in a solution containing $4 \mathrm{X}$ SSC ( $600 \mathrm{mM}$ sodium chloride, $60 \mathrm{mM}$ sodium citrate, and $\mathrm{pH} 7.0$ ) and $0.1 \% \mathrm{SDS}$, pre-heated at $50^{\circ} \mathrm{C}$, and kept at this temperature for $15 \mathrm{~min}$, before rinsing with DI water and drying under a nitrogen stream. Fluorescence images of each chip were obtained using a confocal laser scanner (ScanArray Lite, Perkin Elmer), with the laser power set at 22\% and the photomultiplier tube (PMT) gain at $64 \%$ and analysed using the ScanArray Express software.

\subsection{Interferometric measurements with dual polarization interferometry}

To measure the coating thickness, a silicon oxynitride AnaChip slide was pretreated with oxygen plasma, then inserted into the fluidic compartment of Analight Bio 200 , and kept at $20^{\circ} \mathrm{C}$ under PBS flow. A $1 \% \mathrm{w} / \mathrm{v}$ solution of copoly azide in water was slowly injected into the chip channels at a flow rate of $6 \mu 1 / \mathrm{min}$ for $15 \mathrm{~min}$. The flow was interrupted for
$30 \mathrm{~min}$, to allow the adsorption of the polymer onto the surface. Subsequently, PBS was injected into the channel at a flow rate of $5 \mu 1 / \mathrm{min}$ for $16 \mathrm{~h}$, to rinse the excess of polymers. A standard calibration procedure was conducted before each experiment, using $80 \% \mathrm{w} / \mathrm{v}$ ethanol and MQ water solutions. Subsequently, a solution of copoly DBCO $(1 \% \mathrm{w} / \mathrm{v}$ in water) was injected following the same procedure adopted for the first coating layer: after $15 \mathrm{~min}$ at a flow rate of $6 \mu \mathrm{l} / \mathrm{min}$, the flow was interrupted for 30 minutes and finally the chip was rinsed for 16 hours at $5 \mu \mathrm{l} / \mathrm{min}$. The data collected were analyzed using the Analight Explorer software.

\subsection{Mass density measurements with interferometric reflectance imaging sensor (IRIS)}

The Interferometric reflectance imaging sensor (IRIS) technique is introduced and fully described in $[20,21]$. Briefly, the system takes advantage of the optical properties of layered substrates to detect very small amounts of biomass that accumulate on an active surface, namely, a silicon chip with a thermally grown $110 \mathrm{~nm} \mathrm{SiO}{ }_{2}$ layer. When the chip is illuminated from the top with a coherent source, it creates a common path interferometer. By measuring the reflectance from the substrate at distinct wavelengths across the visible spectrum, it is possible to reconstruct the reflectance curve at the $\mathrm{SiO}_{2}$-biomass interface. Real-time changes in the optical path length due to the capture of a target of interest cause a measurable shift in this reflectance curve.

Practically, illumination from four independent LEDs peaked at four different wavelengths $(455,518,598$ and $635 \mathrm{~nm}$ ) is projected onto the chip through a non-polarizing beam splitter and the chip is subsequently imaged with a CCD camera (PointGrey). The optical setup can be described as a simple reflecting microscope.

To measure the biomass thickness, a four-color IRIS image of the chip was acquired before and after hybridization of the polymeric probe array with the complementary oligonucleotide. The images obtained were analyzed through a custom-made plugin for the imaging software ImageJ to obtain the reflectance values. Further analysis was performed with a custom-written MATLAB analysis software, which works by converting the raw reflectance data to thickness values, normalizing for the background and generating the curves in units of mass per unit 
area. Further detail about this analysis method can be found in [22].

\subsection{Miniaturized multilayer}

\subsubsection{DNA polymeric probe synthesis and functional test}

To a $100 \mu \mathrm{M}$ azido modified COCU8 solution, a solution of copoly DBCO was added to a final polymer concentration of $0.1 \% \mathrm{w} / \mathrm{v}$. The solution was incubated overnight at room temperature and then diluted with printing buffer (sodium phosphate $150 \mathrm{mM}$, $0.01 \%$ sucrose monolaureate) so that the oligonucleotide had the following concentrations: $0.1,0.5$, $1,5,10 \mu \mathrm{M}$. As control, solutions with the same concentration of $\mathrm{NH}_{2} \mathrm{COCU} 8$ and DBCO COCU8 were immobilized directly onto copoly (DMA-NASMAPS) and copoly azide coated silicon slides.

The solutions were then spotted onto the coated surfaces using a noncontact microarray spotter (Scienion sciFLEXARRAYER S12) using a $80 \mu \mathrm{m}$ nozzle. The spot volume, temperature, and humidity were precisely controlled to $400 \mathrm{pl}, 22^{\circ} \mathrm{C}$, and $65 \%$, respectively; spot pitch was set at $350 \mu \mathrm{m}$. Immediately after spotting, all chips were stored overnight in a sealed chamber, saturated with sodium chloride ( $40 \mathrm{~g} / 100 \mathrm{ml} \mathrm{H}_{2} \mathrm{O}$ ). After incubation, only the silicon chips coated with copoly(DMA-NAS-MAPS) were treated with ethanolamine blocking solution $(50 \mathrm{mM}$ in $0.1 \mathrm{M}$ Tris/ $\mathrm{HCl}$ buffer $\mathrm{pH} 9$ ), pre-heated, and kept at $50^{\circ} \mathrm{C}$ for $15 \mathrm{~min}$. The slides coated with the click copolymers did not need this step. Consequently, all chips were rinsed with DI water and immersed in a solution containing 4X SSC ( $600 \mathrm{mM}$ sodium chloride, $60 \mathrm{mM}$ sodium citrate, and $\mathrm{pH} 7.0$ ) and $0.1 \%$ SDS, pre-heated at $50^{\circ} \mathrm{C}$, and kept at this temperature for $15 \mathrm{~min}$, before rinsing with DI water and drying under a nitrogen stream.

Printed chips were then incubated with a complementary oligonucleotide target, 5'-Cy3-TCACTTTTACCTTATAGGTGGGC-3'
(COCU10) tagged with Cy3 for fluorescence detection. COCU10 was diluted to a final concentration of $1 \mu \mathrm{M}$ in an aqueous solution containing $2 \mathrm{X} \mathrm{SSC}$, $0.1 \% \mathrm{SDS}$, and $0.2 \mathrm{mg} / \mathrm{ml}$ of BSA; $15 \mu \mathrm{l}$ of this solution were layered on the hybridization area and covered with a coverslip. The reaction was performed in a humid chamber at $65^{\circ} \mathrm{C}$ for 2 hours, then the chips were washed with a $4 \mathrm{X}$ SSC solution at room temperature (to remove the coverslip), and finally, unbound oligonucleotides were removed using two successive rinses ( 5 min each) with a $2 \mathrm{X} \mathrm{SSC} / 0.1 \%$ SDS solution, pre-warmed at hybridization temperature $\left(65^{\circ} \mathrm{C}\right)$. Other two washes with $0.2 \mathrm{X} \mathrm{SSC}$ and $0.1 \mathrm{X} \mathrm{SSC}$, carried out both at room temperature for $1 \mathrm{~min}$, were then performed, and finally, the slides were dried under a nitrogen stream. Fluorescence images of each chip were obtained using a confocal laser scanner (ScanArray Lite, Perkin Elmer), with the laser power set at $55 \%$ and the photomultiplier tube (PMT) gain at 55\% of their power and analysed using the ScanArray Express software.

\subsubsection{Peptide polymeric probe synthesis}

A polymeric probe was prepared also using peptides. In particular, OMPA peptides were synthesized with an azido or DBCO group as shown in Figure 4.

The peptide OMPA is part of the Outer Membrane Protein A family of proteins and it is an epitope identified from the BPSL2765 antigen of B. Pseudomallei, connected to the survival of the bacteria [23].

These peptides were dissolved to a final concentration of $10 \mathrm{mg} / \mathrm{ml}(3.55 \mathrm{mM})$ in a $50 \% \mathrm{v} / \mathrm{v}$ acetonitrile solution in water.

In order to prepare the peptide polymeric probe, a $0.04 \% \mathrm{w} / \mathrm{v}$ solution of copoly azide or DBCO was prepared (in $25 \mathrm{mM}$ sodium acetate buffer $\mathrm{pH} 4.8$ with $15 \mathrm{mM}$ trehalose) so that the concentration of functional groups of each polymer is $80 \mu \mathrm{M}$. This solution was then diluted $1: 1$ with a $80 \mu \mathrm{M}$ solution of OMPA-N $\mathrm{N}_{3}$ or OMPA-DBCO in $25 \mathrm{mM}$ sodium

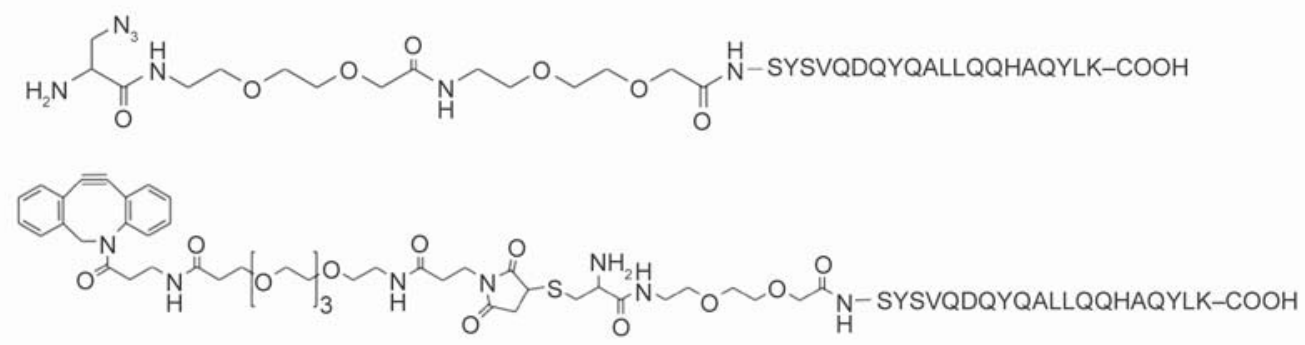

Figure 4. Chemical formulas of the OMPA peptides. 
acetate buffer $\mathrm{pH} 4.8$ with $15 \mathrm{mM}$ trehalose and let react overnight. These solutions were then spotted on copoly azide or copoly DBCO coated slides as previously reported.

\subsubsection{Limit of detection (LOD) evaluation}

Slides coated with copoly azide and copoly DBCO were spotted with the peptide polymeric probe prepared as described in the previous section. Slides were stored overnight and then washed 10 minutes with PBS on a shaker, rinsed quickly with DI water and dried with a nitrogen stream. Each slide was then incubated with increasing concentrations (from 0 to $50 \mathrm{ng} / \mathrm{ml}$ ) of a rabbit polyclonal antibody against OMPA peptide for 2 hours at room temperature in a humid chamber. Slides were washed with washing buffer for 10 minutes on a shaker, rinsed with DI water and dried with a nitrogen stream. Subsequently the slides were incubated with a detection antibody labelled with Cy3 for fluorescent detection $(1 \mu \mathrm{g} / \mathrm{ml})$, washed as previously reported. Slides were scanned for fluorescence detection, the values were fitted with a linear regression and the limits of detection (LOD) were extrapolated from the fluorescent value of blank samples plus 3 standard deviations $(3 \sigma)$.

As a control, non-derivatized OMPA- $\mathrm{N}_{3}$ and OMPADBCO peptides were immobilized directly onto copoly DBCO and copoly azide coated slides respectively. In particular, peptides were dissolved to a final concentration of $10 \mathrm{mg} / \mathrm{ml}(3.55 \mathrm{mM})$ in a $50 \% \mathrm{v} / \mathrm{v}$ acetonitrile solution in water, then diluted to $40 \mu \mathrm{M}$ in $25 \mathrm{mM}$ sodium acetate buffer $\mathrm{pH} 4.8$ with $15 \mathrm{mM}$ trehalose and deposited onto slides using a piezoelectric spotter. These slides were then processed as previously reported.

\section{Results and discussion}

Click chemistry reactions are gaining increasing importance in the development of microarray surfaces because of the peculiar characteristics of high yield, velocity and orthogonality in probe immobilization. Our group has recently introduced a family of well characterized copolymers for surface derivatization that enable click chemistry reactions such as the copper-catalyzed azide-alkyne 1,3-dipolar cycloaddition and the catalyst-free strain-promoted azide-alkyne cycloaddition (SPAAC) [5] obtained by post polymerization modification of a functional and surface adhesive polymer. The coating procedure is very quick and robust and simply consists in immersing the microarray substrate (which can be of different materials) into a diluted water solution of these copolymers. In this work we exploit the click functionalities not only to immobilize probes in a quick and oriented manner, but also to obtain covalently bound, multilayered coatings. Different architectures are investigated, starting from classic extended layers to localized layers by spotting combinations of polymer solutions covalently linked to bioprobes. This strategy offers not only the possibility of increasing the tri-dimensionality of the coating, but it also improves the efficiency of hybridization.

\subsection{Multilayered coating}

In order to obtain covalently bound layers of coatings, the substrate undergoes repeated cycles of coating, using copolymers with complementary click functionalities (see scheme in Figure 2).

First click linked multilayers were obtained using copoly Alkyne (data not shown), but considering that the so called strain-promoted azide-alkyne cycloaddition (SPAAC) does not require addition of catalysts due to the constrained structure of the cyclooctyne of the DBCO groups, copoly Alkyne was replaced by copoly DBCO. As a proof of concept, three polymer layers were obtained as described before, by alternating coatings of copoly DBCO and copoly azide. Azido or DBCO oligonucleotides (depending on the functionality underneath) together with a control amino-modified oligonucleotide were immobilized on the surface of three chips each with alternate azide and DBCO layers (from 1 to 3 ) and then hybridized with a fluorescently labelled, complementary oligonucleotide. As shown in Figure 5, an homogenous coverage of the surface is confirmed, since only specific signals are detected: on the first layer, only the signal corresponding to the DBCO oligonucleotide is observed, whereas on the second layer only the azido-modified oligonucleotide is detected. On the third layer only the signal of DBCO oligonucleotide is visible, but not that of the azide oligonucleotide. The images of Figure 5 demonstrate that the immobilization is due to covalent reaction between complementary functionalities, since no signals are detected where amino modified oligonucleotides are spotted. Furthermore, the intensity of signal resulting from the hybridization of the DBCOoligonucleotide on the third layer does not decrease compared to that on the first layer, suggesting that the density of functional groups in the three layers 

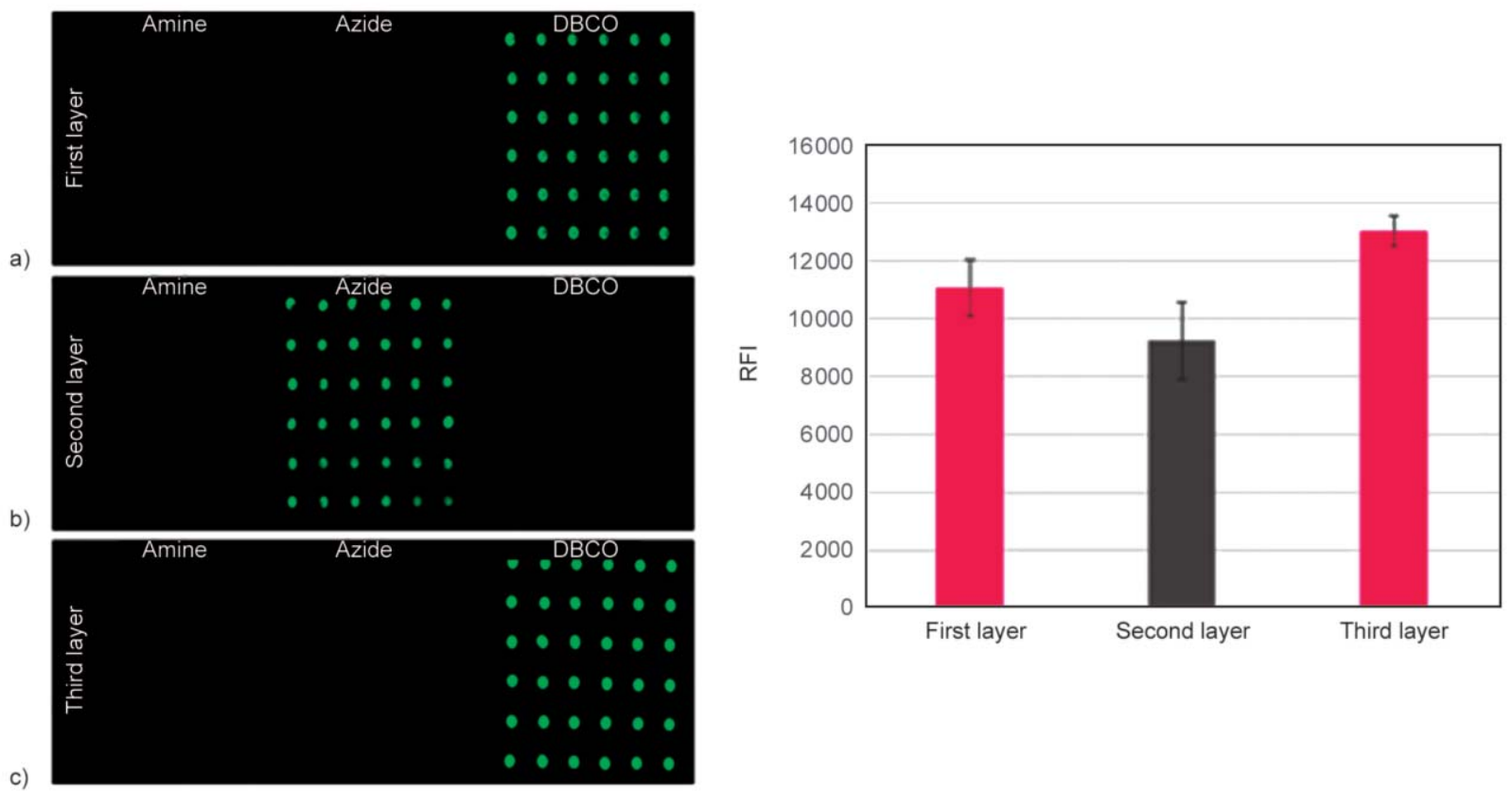

c)
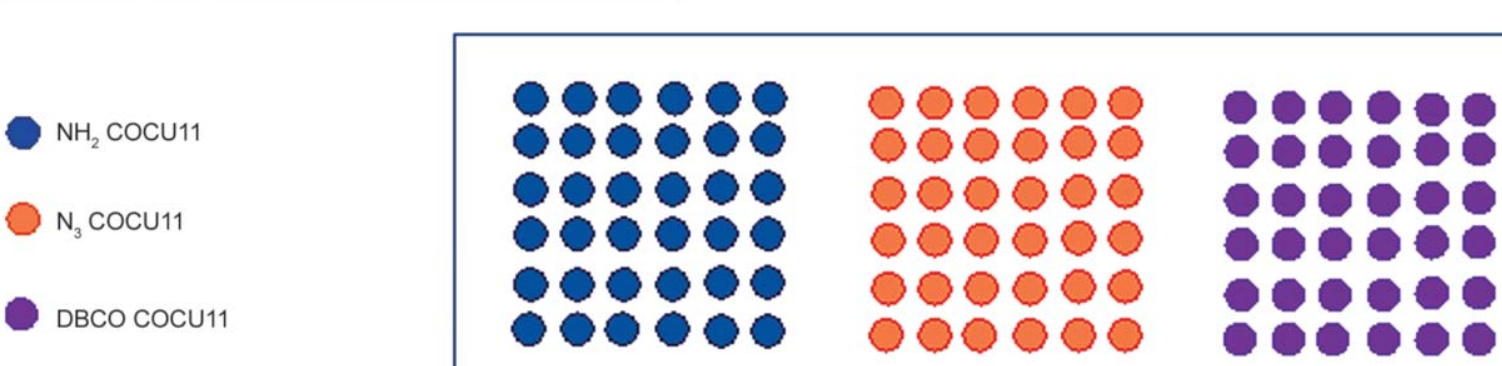

d)

Figure 5. Fluorescence images of glass slides coated with a) first layer of copoly azide, b) a second layer of copoly DBCO, and c) a third layer of copoly azide. Amino, azido, and DBCO modified oligo were spotted at each step to highlight the variation of functionality for layer according to the spotting scheme reported in d). The histogram shows the fluorescence intensity signals obtained for each oligonucleotide hybridized with its complementary strand on each layer. The arrays were scanned using the laser power at 22\% and the PMT gain at $64 \%$.

is similar. The terpolymers used in this work have a silane moiety pending from the backbone that facilitates the binding of contiguous layers through formation of siloxane bridges. To investigate the role of this monomer in the formation of multilayers, a slide with a copoly(DMA-MAPS) layer was coated with an azide copolymer. The inner layer does not present functional groups capable of reacting with azides. However, the azide copolymer did form a film on the surface as demonstrated by the binding of a DBCO fluorescent oligonucleotide spotted on the surface (data not shown). It is possible that silane contributes to promote the binding between different polymers although it is difficult to quantify the number of siloxane bridges within a nanometric layer considering that the molar fraction of the silane is only $1 \%$. Unquestionably, as demonstrated in a previous work, silane is crucial in stabilizing the coating on the surface [3]. Complex experiments that are not within the scope of this work, are needed to investigate the specific contribution of MAPS groups to the binding of polymer layers.

\subsection{Surface characterization via dual polarization interferometry (DPI) and interferometric reflectance imaging sensor (IRIS)}

The thickness, the density, and the mass of the coating layers were evaluated by two different interferometric techniques.

Dual polarization interferometry, allows to measure optical phase changes in an evanescent dual polarization interferometer of biomolecules immobilized onto surface, as comprehensively presented in references $[24,25]$. The coating is formed on the surface of a DPI chip (previously activated by oxygen plasma) by flowing a solution of copoly azide into the chip channels; subsequently, a second layer is 
Table 1. Thickness, mass, and density, measured using DPI, of the first layer of copoly azide and the second layer of copoly DBCO covalently bound to the first one.

\begin{tabular}{|l|c|c|c|}
\hline & $\begin{array}{c}\text { Thickness } \\
{[\mathbf{n m}]}\end{array}$ & $\begin{array}{c}\text { Mass } \\
{\left[\mathbf{n g} / \mathbf{m m}^{\mathbf{2}}\right]}\end{array}$ & $\begin{array}{c}\text { Density } \\
{\left[\mathbf{g} / \mathbf{c m}^{\mathbf{3}}\right]}\end{array}$ \\
\hline First layer & $7.83 \pm 1.02$ & $1.40 \pm 0.34$ & $0.18 \pm 0.05$ \\
\hline Second layer & $15.12 \pm 1.01$ & $2.85 \pm 0.05$ & $0.19 \pm 0.01$ \\
\hline
\end{tabular}

obtained by flowing copoly DBCO, whose functional groups reacted through a SPAAC reaction with the azide groups of the first coating layer. Thickness, mass, and density of the polymeric films are measured while they formed. The data of thickness and mass of the second layer double compared to the first layer demonstrating that the layers of DBCO and azide polymers bind to each other (Table 1). On the contrary, the density remains similar; in fact the two polymers have the same chemical structure, and the interactions with the surface do not alter their compactness. Therefore, the density does not differ when of the polymer layers accumulate on the surface. The measurements are collected after 16 hours of PBS flowing, to demonstrate the high stability of the adsorbed coating on the surface and the covalent interaction between the layers.

IRIS technique allows to measure mass density and morphology of the polymeric probes' spots (described in Section 2.7.1.). This method allows the user to precisely measure the biomass thickness on a layered substrate by evaluating the spectral signature of the surface, as fully described in [21].

To perform these measurements, we spotted two different concentrations of the polymeric probe on a clean IRIS chip: 5 and $10 \mu \mathrm{M}$. We captured the dry image of the chip with the IRIS before the incubation by sampling it at four distinct wavelengths in the visible range (Figure 6). By reconstructing the reflectance curve at every pixel of the image, we were able to convert the reflectance raw data to mass per unit area values, as detailed in [22]. This way,

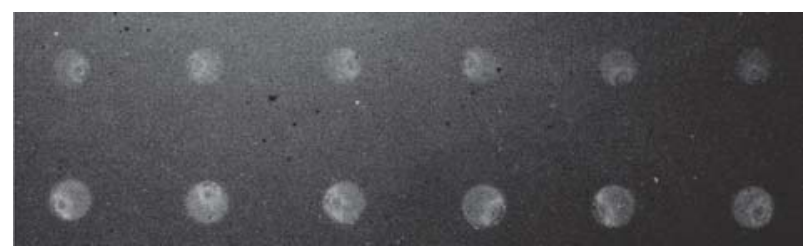

Figure 6. IRIS dry image of the polymeric probe spots before incubation with $1 \mu \mathrm{M}$ COCU10. The upper row was spotted at $5 \mu \mathrm{M}$, while the bottom row was spotted at $10 \mu \mathrm{M}$. From this image, the morphology of the spots can also be observed.
Table 2. Theoretical spotted mass density vs. actual immobilized mass density, measured using IRIS, of the polymeric probe spots at 5 and $10 \mu \mathrm{M}$ before incubation with the complementary oligonucleotide. The approximate percentage of the immobilized mass relative to the ideal spotted mass value is shown in parentheses.

\begin{tabular}{|l|c|c|}
\hline & $\begin{array}{c}\text { Spotted mass } \\
\text { density } \\
{\left[\mathbf{n g} / \mathbf{m m}^{\mathbf{2}}\right]}\end{array}$ & $\begin{array}{c}\text { Measured mass } \\
\text { density } \\
{\left[\mathbf{n g} / \mathbf{m m}^{\mathbf{2}}\right]}\end{array}$ \\
\hline Polymeric probe $\quad 5 \mu \mathrm{M}$ & $2.68 \pm 0.01$ & $0.61 \pm 0.02(\approx 23 \%)$ \\
\hline Polymeric probe $10 \mu \mathrm{M}$ & $3.38 \pm 0.01$ & $1.93 \pm 0.04(\approx 57 \%)$ \\
\hline
\end{tabular}

Table 3. Mass density, measured using IRIS, of the polymeric probe spots at 5 and $10 \mu \mathrm{M}$ before and after incubation with the complementary oligonucleotide COCU10 at $1 \mu \mathrm{M}$.

\begin{tabular}{|l|c|c|}
\hline & $\begin{array}{c}\text { Mass before } \\
\text { hybridization } \\
{\left[\mathbf{n g} / \mathbf{m m}^{\mathbf{2}}\right]}\end{array}$ & $\begin{array}{c}\text { Mass after } \\
\text { hybridization } \\
{\left[\mathbf{n g} / \mathbf{m m}^{\mathbf{2}}\right]}\end{array}$ \\
\hline Polymeric probe $5 \mu \mathrm{M}$ & $0.61 \pm 0.02$ & $0.71 \pm 0.01$ \\
\hline Polymeric probe $10 \mu \mathrm{M}$ & $1.93 \pm 0.04$ & $2.11 \pm 0.01$ \\
\hline
\end{tabular}

we were able to compare the initial spotted mass values with the actual immobilized mass. The theoretical values for the spotted mass were obtained by calculating and summing up the polymer and the oligonucleotide mass that are present in a $400 \mathrm{pl}$ droplet - that is, the size of the droplet used for spotting (Table 2). The data presented in Table 2 show that a good fraction of the spotted mass is immobilized onto the surface. This is a reasonable estimate of the spotting efficiency of this probe.

The chip was then incubated with the complementary oligonucleotide $(1 \mu \mathrm{M})$ to allow hybridization. The incubation was performed under flow for 30 minutes. After washing the chip with filtered PBS, a new IRIS measurement was performed, allowing us to compare the deposited mass before and after hybridization (Table 3). The data presented in Table 3 suggest that having a higher concentration of probes on the surface increases the hybridization efficiency, which is in agreement with the results shown in paragraph 3.3.

\subsection{Immobilization of an oligonucleotide bound to a polymer: polymeric probe}

An alternative approach to multilayer formation is through the spotting of probes covalently bound to the copolymers discussed above. The peculiar characteristics of water solubility and high reactivity rate of the click reaction between DBCO and azido groups allow to generate micro-functionalized areas 
by spotting what we named a polymeric probe, obtained by conjugating the polymer (copoly azide or copoly DBCO) with the probe that needs to be immobilized on the surface (see Figure 3).

To demonstrate this concept, we added to the usual spotting solution, consisting of a fluorescently labeled azido-modified probe, increasing amounts of copoly DBCO whose click groups react in part with the azido probe and in part with the azido groups on the surface of the coated slide. A deep optimization of polymer concentration and time required to bind the probe to the polymer chains was carried out. Based on these results, the hybridization efficiency of this system was tested using a polymeric probe obtained by dissolving the two reagents (copoly DBCO and an azido modified oligonucleotide) in such a way that the moles of the polymer functional groups are 2-fold the moles of the probe to be bound. In this way, half of the DBCO groups binds the probes, while the remaining half interacts with the complementary azido groups of the coated surface. As a control, on copoly azide and copoly (DMANAS-MAPS) coated surfaces, an oligonucleotide with the appropriate modification (DBCO or amine) is directly immobilized. Figure 7 compares the fluorescence signals obtained after hybridization of each array with the fluorescently labelled complementary oligonucleotide $(1 \mu \mathrm{M})$.

In general, the immobilization of the probes through click reactions leads to fluorescence intensity signals higher than those obtained on surfaces coated with copoly (DMA-NAS-MAPS). These results suggest

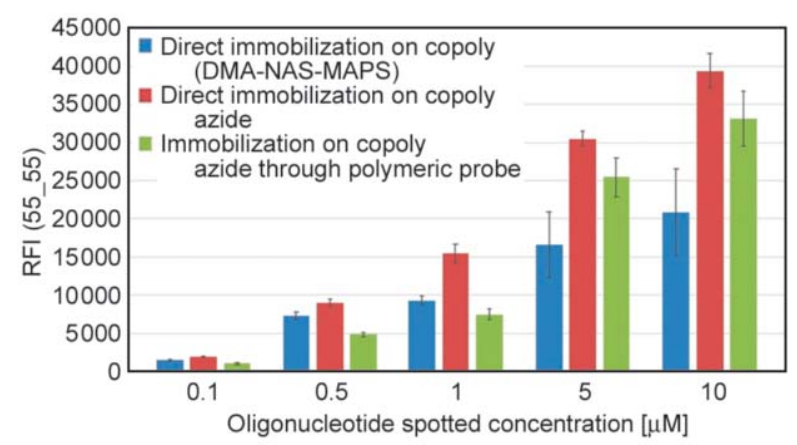

Figure 7. Fluorescence intensity signals obtained by hybridization with a fluorescently labelled oligonucleotide $(1 \mu \mathrm{M})$ complementary to that deposited on the surface. In particular, an oligonucleotide is immobilized either directly on surfaces coated with copoly azide and copoly (DMA-NAS-MAPS) or through the formation of a polymeric probe. Values were obtained by setting the laser power to $55 \%$ and the PMT to $55 \%$. that on surfaces coated with click polymers, not only the probe is able to effectively bind to the surface in a stable manner, but also that increasing the density of the immobilized probes on the surface improves hybridization performance.

\subsection{Peptide polymeric probe synthesis and limit of detection (LOD) evaluation}

The approach described so far to obtain DNA polymeric probes, can be applied also to peptides, to increase their accessibility by the target. The use of peptides instead of proteins in microarray technique offers several advantages such as, for example, a high degree of synthetic flexibility, ease of handling and the greater stability of the immobilized probes. However, a correct probe immobilization and exposure becomes particularly decisive in the case of peptides: small molecules $(3-10 \mathrm{kDa})$ that once immobilized on the surface are designed to capture molecular counterparts, such as antibodies, which are much larger $(150 \mathrm{kDa})$.

As a proof of concept, we have obtained a polymeric probe by reacting a DBCO-modified peptide with copoly azide. In particular, the peptide we have chosen, defined OMPA3, is an epitope identified from the BPSL2765 antigen of $B$. pseudomallei by Peri et al. [26]. This antigen is part of the OMPA (Outer Membrane Protein A) family of proteins, a group of genetically related proteins responsible for bacterial survival. Previous studies have validated peptide OMPA3 as a diagnostic probe for Burkholderia infections in subjects affected by cystic fibrosis [26] and investigated the effectiveness of different peptide-to-surface conjugation strategies [17]. We found that all the click chemoselective reactions that were evaluated, i.e. thiol-maleimide, CuAAC and SPAAC, showed a comparable efficiency in terms of final diagnostic performance, even if some differences may direct the selection of one strategy over another one. Specifically, the binding efficiency to microarray surfaces was the highest when using azide-alkynecycloaddition (both in the $\mathrm{Cu}$-catalyzed and $\mathrm{Cu}$-free versions), however, immobilization using SPAAC resulted in more homogeneous spots with respect to the $\mathrm{Cu}$-catalyzed version, highlighting that the presence of the metal catalyst may somehow negatively affect the spots shape. Based on these previous finding, a functional assay was developed on slides coated with copoly azide or copoly DBCO on which OMPA3 was immobilized by SPAAC directly or 
through the formation of a polymeric probe, obtained as described in Section 2.7.1.

To calculate the lowest detection limit (LOD) of the two systems, two calibration curves were built by incubating the immobilized peptide with increasing concentrations (from 0 to $50 \mathrm{ng} / \mathrm{ml}$ ) of an antibody against OMPA3. To reveal the interaction between the peptide and its antibody a second incubation with a Cy3 labelled secondary antibody was performed and fluorescence signals were plotted with a linear regression to calculate the limits of detection (LOD). LOD was extrapolated from the fluorescent value of blank samples plus 3 standard deviations ( $3 \sigma)$. A typical curve reporting average fluorescence intensity of 64 spots incubated at 5 different concentrations of antibody, is shown in Figure 8. Table 4 reports the LOD values calculated for the two different immobilization approaches (direct vs polymeric probe) on copoly azide or copoly DBCO coated slides.

The LOD values obtained through the immobilization of the polymeric probe, are significantly lower than those obtained in the case of direct immobilization of the peptide. Very likely, the deposition of one polymer on top of another one (the coating of the surface) and the formation of covalent bonds between the two by click chemistry, allows to expand the surface three-dimensionally in such a way that the peptide is far from the surface and better exposed towards the reaction environment. This phenomenon facilitates the recognition of the peptide by the antibody in solution resulting in an increased affinity between the two molecules that can be exploited to

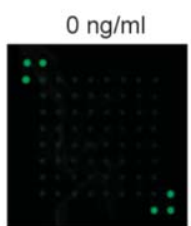

$10 \mathrm{ng} / \mathrm{ml}$

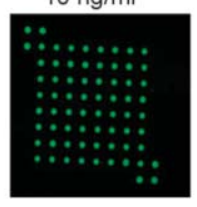

a)
Table 4. LOD values calculated for the direct or through the polymeric probe approach immobilization of OMPA3 peptide on copoly azide or copoly DBCO coated slides.

\begin{tabular}{|l|r|}
\hline \multicolumn{1}{|c|}{ Immobilization type } & $\begin{array}{c}\text { LOD } \\
\text { [pg/l] }\end{array}$ \\
\hline Direct on copoly azide & 688 \\
\hline Polymeric probe on copoly azide & 29 \\
\hline Direct on copoly DBCO & 613 \\
\hline Polymeric probe on copoly DBCO & 38 \\
\hline
\end{tabular}

increase the sensitivity of serological assays in diagnostic applications.

\section{Conclusions}

In conclusion, we have exploited the characteristics of copolymers bearing complementary click groups to obtain different types of covalently linked multilayered coatings and 3D-architectures. The proposed strategies offer not only the possibility to expand the thickness of the coating, which remains stable and functional, but also provides the possibility to bind to the surface probes that otherwise would not be immobilized because not reactive with the surface. In addition, the proposed configuration allows to bind the probes through a polymeric linker, thus spacing them from the surface and reducing surface effects on hybridization efficiency.

\section{References}

[1] Dufva M.: Fabrication of high quality microarrays. Biomolecular Engineering, 22, 173-184 (2005). https://doi.org/10.1016/j.bioeng.2005.09.003

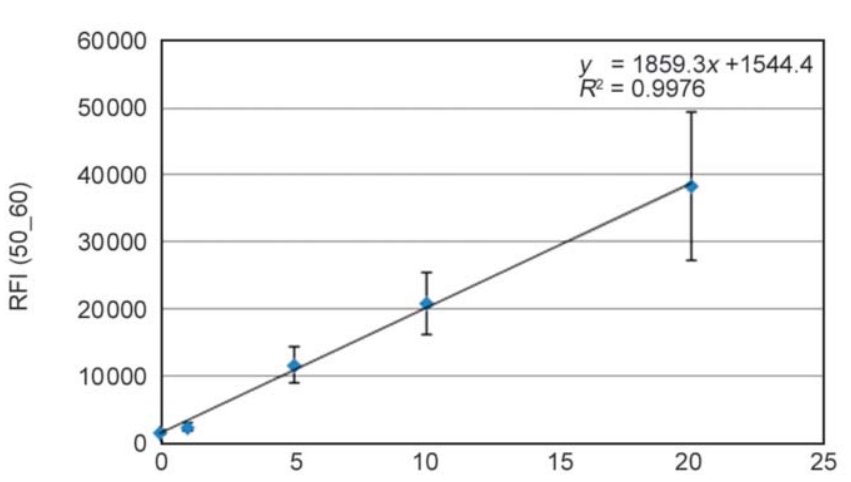

b)

$[\mathrm{ng} / \mathrm{ml}] \mathrm{Ab}$

Figure 8. a) Fluorescence images of slides coated with copoly DBCO, spotted with OMPA3 peptide and incubated with increasing concentrations of an antibody against OMPA peptide; slides were scanned with a confocal laser scanner with power set at 50\% and PMT set at $60 \%$ and mean values of each array were plotted to obtain a calibration curve (b). The limit of detection (LOD) was extrapolated from the fluorescent value of blank samples plus 3 standard deviations. 
[2] Sonawane M. D., Nimse S. B.: Surface modification chemistries of materials used in diagnostic platforms with biomolecules. Journal of Chemistry, 2016, 9241378/1-9241378/19 (2016).

https://doi.org/10.1155/2016/9241378

[3] Pirri G., Damin F., Chiari M., Bontempi E., Depero L. E.: Characterization of a polymeric adsorbed coating for DNA microarray glass slides. Analytical Chemistry, 76, 1352-1358 (2004).

https://doi.org/10.1021/ac0352629

[4] Zilio C., Sola L., Damin F., Faggioni L., Chiari M.: Universal hydrophilic coating of thermoplastic polymers currently used in microfluidics. Biomedical Microdevices, 16, 107-114 (2014).

https://doi.org/10.1007/s10544-013-9810-8

[5] Sola L., Damin F., Gagni P., Consonni R., Chiari M.: Synthesis of clickable coating polymers by postpolymerization modification: Applications in microarray technology. Langmuir, 32, 10284-10295 (2016).

https://doi.org/10.1021/acs.langmuir.6b02816

[6] Gauthier M. A., Gibson M. I., Klok H-A.: Synthesis of functional polymers by post-polymerization modification. Angewandte Chemie International Edition, 48, 48-58 (2009).

https://doi.org/10.1002/anie.200801951

[7] Sedlacek O., Janouskova O., Verbraeken B., Hoogenboom R.: Straightforward route to superhydrophilic poly(2-oxazoline)s via acylation of well-defined polyethylenimine. Biomacromolecules, 20, 222-230 (2019). https://doi.org/10.1021/acs.biomac.8b01366

[8] Kubo T., Easterling C. P., Olson R. A., Sumerlin B. S.: Synthesis of multifunctional homopolymers via sequential post-polymerization reactions. Polymer Chemistry, 9, 4605-4610 (2018).

https://doi.org/10.1039/C8PY01055B

[9] Qamhieh K., Pettitt B. M.: Controlling microarray DNA hybridization efficiency by probe-surface distance and external surface electrostatics. AIP Conference Proceedings, 1653, 020090/1-020090/10 (2015).

https://doi.org/10.1063/1.4914281

[10] Katayama H., Ishihama Y., Asakawa N.: Stable capillary coating with successive multiple ionic polymer layers. Analytical Chemistry, 70, 2254-2260 (1998). https://doi.org/10.1021/ac9708755

[11] Wei F., Ye F., Li S., Wang L., Li J., Zhao G.: Layer-bylayer coating of chitosan/pectin effectively improves the hydration capacity, water suspendability and tofu gel compatibility of okara powder. Food Hydrocolloids, 77, 465-473 (2018).

https://doi.org/10.1016/j.foodhyd.2017.10.024

[12] Parbat D., Manna U.: Synthesis of 'reactive' and covalent polymeric multilayer coatings with durable superoleophobic and superoleophilic properties under water. Chemical Science, 8, 6092-6102 (2017).

https://doi.org/10.1039/C7SC01055A
[13] Seo J., Schattling P., Lang T., Jochum F., Nilles K., Theato P., Char K.: Covalently bonded layer-by-layer assembly of multifunctional thin films based on activated esters. Langmuir, 26, 1830-1836 (2010). https://doi.org/10.1021/la902574z

[14] Yang W. J., Pranantyo D., Neoh K-G., Kang E-T., Teo S. L-M., Rittschof D.: Layer-by-layer click deposition of functional polymer coatings for combating marine biofouling. Biomacromolecules, 13, 2769-2780 (2012). https://doi.org/10.1021/bm300757e

[15] Such G. K., Quinn J. F., Quinn A., Tjipto E., Caruso F.: Assembly of ultrathin polymer multilayer films by click chemistry. Journal of the American Chemical Society, 128, 9318-9319 (2006).

https://doi.org/10.1021/ja063043+

[16] Landi F., Johansson C. M., Campopiano D. J., Hulme A. N.: Synthesis and application of a new cleavable linker for 'click'-based affinity chromatography. Organic and Biomolecular Chemistry, 8, 56-59 (2010). https://doi.org/10.1039/B916693A

[17] Gori A., Sola L., Gagni P., Bruni G., Liprino M., Peri C., Colombo G., Cretich M., Chiari M.: Screening complex biological samples with peptide microarrays: The favorable impact of probe orientation via chemoselective immobilization strategies on clickable polymeric coatings. Bioconjugate Chemistry, 27, 2669-2677 (2016). https://doi.org/10.1021/acs.bioconjchem.6b00426

[18] Sola L., Chiari M.: Modulation of electroosmotic flow in capillary electrophoresis using functional polymer coatings. Journal of Chromatography A, 1270, 324-329 (2012). https://doi.org/10.1016/j.chroma.2012.10.039

[19] Relógio P., Charreyre M-T., Farinha J. P. S., Martinho J. M. G., Pichot C.: Well-defined polymer precursors synthesized by RAFT polymerization of $N, N$-dimethylacrylamide/ $N$-acryloxysuccinimide: Random and block copolymers. Polymer, 45, 8639-8649 (2004). https://doi.org/10.1016/j.polymer.2004.10.056

[20] Özkumur E., Needham J. W., Bergstein D. A., Gonzalez R., Cabodi M., Gershoni J. M., Goldberg B. B., Ünlü M. S.: Label-free and dynamic detection of biomolecular interactions for high-throughput microarray applications. Proceedings of the National Academy of Sciences of the United States of America, 105, 7988-7992 (2008). https://doi.org/10.1073/pnas.0711421105

[21] Özkumur E., Yalçin A., Cretich M., Lopez C., Bergstein D., Goldberg B. B., Chiari M., Ünlü M. S.: Quantification of DNA and protein adsorption by optical phase shift. Biosensors and Bioelectronics, 25, 167-172 (2009).

https://doi.org/10.1016/j.bios.2009.06.033

[22] Sevenler D., Ünlü M. S.: Numerical techniques for highthroughput reflectance interference biosensing. Journal of Modern Optics, 63, 1115-1120 (2016).

https://doi.org/10.1080/09500340.2015.1117668 
[23] Gourlay L. J., Peri C., Ferrer-Navarro M., ConchilloSolé O., Gori A., Rinchai D., Thomas R. J., Champion O. L., Michell S. L., Kewcharoenwong C., Nithichanon A., Lassaux P., Perletti L., Longhi R., Lertmemongkolchai G., Titball R. W., Daura X., Colombo G., Bolognesi M.: Exploiting the burkholderia pseudomallei acute phase antigen BPSL2765 for structure-based epitope discovery/design in structural vaccinology. Chemistry and Biology, 20, 1147-1156 (2013). https://doi.org/10.1016/j.chembiol.2013.07.010

[24] Swann M. J., Peel L. L., Carrington S., Freeman N. J.: Dual-polarization interferometry: An analytical technique to measure changes in protein structure in real time, to determine the stoichiometry of binding events, and to differentiate between specific and nonspecific interactions. Analytical Biochemistry, 329, 190-198 (2004).

https://doi.org/10.1016/j.ab.2004.02.019
[25] Cross G. H., Reeves A. A., Brand S., Popplewell J. F., Peel L. L., Swann M. J., Freeman N. J.: A new quantitative optical biosensor for protein characterisation. Biosensors and Bioelectronics, 19, 383-390 (2003). https://doi.org/10.1016/S0956-5663(03)00203-3

[26] Peri C., Gori A., Gagni P., Sola L., Girelli D., Sottotetti S., Cariani L., Chiari M., Cretich M., Colombo G.: Evolving serodiagnostics by rationally designed peptide arrays: The Burkholderia paradigm in cystic fibrosis. Scientific Reports, 6, 32873/1-32873/11 (2016). https://doi.org/10.1038/srep32873 